$\underline{\underline{\beta}}=\mathbf{Z}$

\title{
Quantum gravitational applications of nuclear, atomic and astrophysical phenomena
}

\author{
U. V. S. Seshavatharam ${ }^{12} *$ S. Lakshminarayana ${ }^{3}$ \\ ${ }^{1}$ ORNNOVA Technologies India Pvt. Ltd, 11th Cross, Indira Towers, Wilson Gardens, Bangalore-27, India \\ ${ }^{2}$ Honorary faculty, I-SERVE, Alakapuri, Hyderabad-35,AP, India \\ ${ }^{3}$ Dept. of Nuclear Physics, Andhra University, Visakhapatnam-03,AP \\ *Corresponding author E-mail:seshavatharam.uvs@gmail.com
}

\begin{abstract}
By following the old concept of "gravity is having a strong coupling at nuclear scale" and considering the 'reduced Planck's constant' as a characteristic quantum gravitational constant, in this letter we suggest that: 1) There exists a gravitational constant associated with strong interaction, $\mathrm{G}_{\mathrm{s}} \sim 3.328 \times 10^{28} \mathrm{~m}^{3} / \mathrm{kg} / \mathrm{sec}^{2}$. 2) There also exists a gravitational constant associated with electromagnetic interaction, $\mathrm{G}_{\mathrm{e}} \sim 2.376 \times 10^{37} \mathrm{~m}^{3} / \mathrm{kg} / \mathrm{sec}^{2}$. Based on these two assumptions, in a quantum gravitational approach, an attempt is made to understand the basics of final unification with various semi empirical applications like melting points of elementary particles, strong coupling constant, proton-electron mass ratio, proton-neutron stability, nuclear binding energy, neutron star's mass and radius, Newtonian gravitational constant, Avogadro number and molar mass unit. With further research and investigation, a practical model of 'quantum gravitational string theory' can be developed.
\end{abstract}

Keywords: Quantum Gravity; Strong Interaction; Electromagnetic Interaction; Newtonian Gravitational Constant, Schwarzschild Interaction Strength, Neutron Star, Avogadro number and Molar Mass Unit.

\section{Introduction}

Even though 'String theory' and 'Quantum gravity' models[1], [2] are having a strong mathematical back ground and sound physical basis, both the models are failing in understanding the role of the Newtonian gravitational constant [3-8] in atomic and nuclear physics and thus seem to fail in developing a 'workable' model of final unification.

According to W. Lerche: "The most dramatic extension of the Standard Model of particle physics that has been proposed so far is string theory. However, as we will discuss in more detail below, string theory too does not provide very concrete answers to the questions posed above. But what string theory does is to provide a resolution of conceptual problems that are on a far deeper level than these "practical" problems. One of the most important problems in modern theoretical physics is the apparent mutual incompatibility of quantum mechanics and general relativity (the theory of gravity) - one theory describing well the world at very short, the other at long distances. Certainly a truly satisfying unified theory should incorporate the gravitational interaction as well, even though traditionally it is not considered as belonging to particle physics".

According to Juan M. Maldacena: "We now have a theory, called string theory (or M-theory), which has been able already to provide a solution to the first two challenges. Unfortunately, we do not know yet how to solve the third challenge. May be string theory is the solution and we just have to understand it better or maybe we have to modify it in some way. String theory is a theory under construction. We know several limits and aspects of the theory, but we still do not know the fundamental axioms of the theory that would enable us to approach the third challenge" (To Explain the Big Bang and the parameters of the Standard Model).

According to Roberto Onofrio [9], [10], weak interactions are peculiar manifestations of quantum gravity at the Fermi scale, and that the Fermi coupling constant is related to the Newtonian constant of gravitation. In his opinion, at atto-meter scale, Newtonian gravitational constant seems to reach a magnitude of $8.205 \times 10^{22} \mathrm{~m}^{3} \mathrm{~kg}^{-1} \mathrm{sec}^{-2}$. in this context; one can see plenty of papers on 'strong gravity' in physics literature [12-28]. It may be noted that, till date, 'strong gravity' is a non-mainstream theoretical approach to Color confinement/particle confinement having both a cosmological scale and a particle scale gravity. In between $\sim(1960$ to 2000), it was taken up as an alternative to the then young QCD theory by several theorists, including Abdus Salam. Very interesting point to be noted is that, Abdus Salam showed that the 'particle level gravity approach' can produce confinement and asymptotic freedom while not requiring a force behavior differing from an inverse-square law, as does QCD.

In pursuit of bridging the gap in between 'General theory of relativity' and 'Quantum field theory' - in the earlier publications [29-37], the authors proposed three basic assumptions. The authors strongly encourage the readers to go through the above cited references. It may be noted that, in the earlier publications, the authors suggested and validated the role of two gravitational constants associated with strong and electromagnetic interactions. In an integrated approach the authors also showed that, 'quantum of angular momentum' is a characteristic result of the combined effects of gravitational constants associated with proton and electron. In this letter the authors compiled important characteristic relations for good understanding, better accuracy and best presen- 
tation. Each relation seems to have its own characteristic inner meaning.

\section{Two basic assumptions of final unification}

In the earlier publications [29-37] the authors proposed and established the following two assumptions. For detailed information readers are strongly encouraged to see the references.

Assumption-1:Magnitude of the gravitational constant associated with the electromagnetic interaction is,

$G_{e} \approx 2.376 \times 10^{37} \mathrm{~m}^{3} \mathrm{~kg}^{-1} \mathrm{sec}^{-2}$.

Assumption-2: Magnitude of the gravitational constant associated with the strong interaction is,

$G_{S} \approx 3.328 \times 10^{28} \mathrm{~m}^{3} \mathrm{~kg}^{-1} \mathrm{sec}^{-2}$.

Note: It may be noted that, with reference to the operating force magnitudes, protons and electrons cannot be considered as 'black holes'. But electrons and protons can be assumed to follow the relations that black holes generally believed to follow. Clearly speaking, in the study of black holes, Newtonian gravitational constant $G_{N}$ plays a major role, whereas in the study of elementary particles, $G_{s}$ and $G_{e}$ play the key role. For detailed information, see the following sub section.

\subsection{Key points to be noted}

1) If it is true that $c$ and $G_{N}$ are fundamental physical constants, then $\left(c^{4} / G_{N}\right)$ can be considered as a fundamental compound constant related to a characteristic limiting force [38-41].

2) Black holes are the ultimate state of matter's geometric structure.

3) Magnitude of the operating force at the black hole surface is of the order of $\left(c^{4} / G_{N}\right)$.

4) Gravitational interaction taking place at black holes can be called as 'Schwarzschild interaction'.

5) Strength of 'Schwarzschild interaction' can be assumed to be unity.

6) Strength of any other interaction can be defined as the ratio of operating force magnitude and the classical or astrophysical force magnitude $\left(c^{4} / G_{N}\right)$.

7) If one is willing to represent the magnitude of the operating force as a fraction of $\left(c^{4} / G_{N}\right)$ i.e. $X$ times of $\left(c^{4} / G_{N}\right)$, where $X<<1$, then

$$
\frac{X \text { times of }\left(c^{4} / G_{N}\right)}{\left(c^{4} / G_{N}\right)} \cong X \rightarrow \text { Effective } G \Rightarrow \frac{G_{N}}{X}
$$

If $X$ is very small, $\frac{1}{X}$ becomes very large. In this way, $X$ can be called as the strength of interaction. Clearly speaking, strength of any interaction is $\frac{1}{X}$ times less than the 'Schwarzschild interaction' and effective $G$ becomes $\frac{G}{X}$.
8) With reference to Schwarzschild interaction, for electromagnetic interaction, $X \approx 2.811 \times 10^{-48}$ and for strong interaction, $X \cong 2.0 \times 10^{-39}$.

9) Characteristic operating force corresponding to electromagnetic interaction is $\left(c^{4} / G_{e}\right) \approx 3.4 \times 10^{-4} \mathrm{~N}$ and characteristic operating force corresponding to strong interaction is $\left(c^{4} / G_{s}\right) \approx 242600 \mathrm{~N}$.

10) Characteristic operating power corresponding to electromagnetic interaction is $\left(c^{5} / G_{e}\right) \approx 101920 \mathrm{~J} / \mathrm{sec}$ and characteristic operating power corresponding to strong interaction is $\left(c^{5} / G_{S}\right) \approx 7.27 \times 10^{13} \mathrm{~J} / \mathrm{sec}$

11) Based on these concepts, it is possible to assume that,

$$
\begin{aligned}
& \frac{\left(m_{e} c^{2}\right)^{\frac{3}{2}}\left(m_{p} c^{2}\right)^{\frac{1}{2}}}{\sqrt{\left(c^{4} / G_{e}\right)\left(c^{4} / G_{s}\right)}} \cong \hbar c \\
& \frac{\left(m_{e} c^{2}\right)^{\frac{3}{2}}\left(m_{p} c^{2}\right)^{\frac{1}{2}}}{\sqrt{\left(c^{5} / G_{e}\right)\left(c^{5} / G_{s}\right)}} \cong \hbar
\end{aligned}
$$

12) As

$$
\left[\left(c^{4} / G_{e}\right),\left(c^{4} / G_{s}\right)\right]<<\left(c^{4} / G_{N}\right)
$$
$\left[\left(c^{5} / G_{e}\right),\left(c^{5} / G_{s}\right)\right]<<\left(c^{5} / G_{N}\right)$, protons and electrons cannot be considered as 'black holes', but may be assumed to follow similar relations that black holes generally believed to follow.

13) According to S.W. Hawking [42], temperature of black hole takes the following expression.

$$
T_{B} \cong \frac{\hbar c^{3}}{8 \pi G_{N} k_{B} M_{B}}
$$

Where $M_{B}$ and $T_{B}$ represent the mass and temperature of a black hole respectively.

According to Abhas Mithra [43], [44], currently believed 'black holes' area kind of "Eternally Collapsing Objects". The so-called massive Black Hole Candidates (BHCs) must be quasi-black holes rather than exact black holes and during preceding gravitational collapse, entire mass energy and angular momentum of the collapsing objects must be radiated away before formation of exact mathematical black holes. Abhas Mitra's peer reviewed papers describe why continued physical gravitational collapse should lead to formation of ECOs rather than true black holes, and the mathematical "black hole" states can be achieved only asymptotically. An ECO is essentially a quasi-stable ultra-compact ball of fire (plasma) which is so hot due to preceding gravitational contraction that its outward radiation pressure balances its inward pull of gravity. Some astrophysicists claimed to have verified this prediction that astrophysical Black Hole Candidates are actually ECOs rather than true mathematical black holes. One can find relevant information at http://www.cv.nrao.edu/tuna/past/2006/NEW_QSO_STRUCTUR E_FOUND.pdf. By considering these two views and by considering the proposed views, melting temperature of elementary particles can be estimated very easily. 
3. Role of the Newtonian gravitational constant in nuclear physics

a) To understand the Proton rest mass

$m_{p} \cong\left(\frac{G_{N}}{G_{e}}\right)^{\frac{1}{6}} \sqrt{M_{p l} m_{e}}$

where, $M_{p l} \cong \sqrt{\frac{\hbar c}{G_{N}}}$

b) To understand the excited levels of proton

$$
m_{x} \cong\left(\frac{G_{N}}{G_{e}}\right)^{\frac{1}{6}}\left(\frac{(n \hbar) m_{e}^{2}}{G_{N}}\right)^{\frac{1}{4}} \cong(n)^{\frac{1}{4}} \times m_{p}
$$

where, $n=1,2,3, .$.

For, $n=1, m_{x} \cong 938.3 \mathrm{MeV} / c^{2}$

For, $n=2, m_{x} \cong 1115.8 \mathrm{MeV} / c^{2}$

For, $n=3, m_{x} \cong 1234.8 \mathrm{MeV} / c^{2}$

For, $n=4, m_{x} \cong 1326.8 \mathrm{MeV} / c^{2}$

For, $n=5, m_{x} \cong 1403.3 \mathrm{MeV} / c^{2}$

For, $n=6, m_{x} \cong 1468.5 \mathrm{MeV} / c^{2}$

For, $n=7, m_{x} \cong 1526.1 \mathrm{MeV} / c^{2}$

For, $n=8, m_{x} \cong 1578.0 \mathrm{MeV} / c^{2}$

For, $n=9, m_{x} \cong 1625.2 \mathrm{MeV} / c^{2}$

For, $n=10, m_{x} \cong 1668.6 \mathrm{MeV} / c^{2}$

These estimated levels assumed to be associated with proton can be compared with currently believed nucleon resonances up to some extent [45]. Extending this idea, other baryonic masses can also be estimated and the authors are working on this.

Based on relation (6), $G_{N}$ can be estimated with the following relation.

$G_{N} \cong\left\{\left(\frac{m_{e}}{m_{p}}\right)^{12}\left(\frac{G_{s} m_{p}^{2}}{\hbar c}\right)\right\} G_{s}$

c) To understand the strong coupling constant

$$
\alpha_{s} \cong\left(\frac{G_{e}}{G_{N}}\right)^{\frac{1}{6}}\left(\frac{G_{s}}{G_{e}}\right) \cong \frac{G_{s}}{G_{N}^{1 / 6} G_{e}^{5 / 6}}
$$

4. To estimate the gravitational constant assumed to be connected with proton

Nuclear unit charge radius: It can be understood as follows [46], [47]

$R_{0} \cong \frac{2 G_{s} m_{p}}{c^{2}}$

Based on relation (9), $G_{S}$ can be estimated with the following relation.

$$
G_{s} \cong \frac{c^{2} R_{0}}{2 m_{p}}
$$

\section{To estimate the gravitational constant as- sumed to be connected with electron}

Ratio of rest mass of proton and electron: It can be understood as follows.

$$
\left(\frac{m_{p}}{m_{e}}\right) \cong\left(\frac{G_{e} m_{e}^{2}}{\hbar c}\right)\left(\frac{G_{s} m_{p}^{2}}{\hbar c}\right)
$$

Thus, based on relation (11), $G_{e}$ can be estimated with the following relation.

$G_{e} \cong\left(\frac{\hbar^{2} c^{2}}{G_{s} m_{e}^{3} m_{p}}\right) G_{s} \cong \frac{2 \hbar^{2}}{m_{e}^{3} R_{0}}$

\section{To estimate the magnitudes of ${ }^{\left(G_{s}, G_{e}, G_{N}, \alpha_{s}\right)}$}

Based on the reference [45]

Let,

$\left\{\begin{array}{l}e \cong 1.602176565(35) \times 10^{-19} \mathrm{C}, \\ \varepsilon_{0} \cong 8.854187817 \times 10^{-19} \mathrm{~F} / \mathrm{m} \\ m_{n} \cong 1.674927471(21) \times 10^{-27} \mathrm{~kg}, \\ m_{p} \cong 1.672621777(74) \times 10^{-27} \mathrm{~kg} \\ m_{e} \cong 9.10938291(40) \times 10^{-31} \mathrm{~kg}, \\ \hbar \cong 1.054571726(47) \times 10^{-34} \mathrm{~J} . \mathrm{sec}\end{array}\right.$

If $R_{0} \cong(1.2384$ to 1.2388$) \mathrm{fm}$,

$G_{S} \cong(3.327167052$ to 3.328241718$) \times 10^{28} \mathrm{~m}^{3} \mathrm{~kg}^{-1} \mathrm{sec}^{-2}$

$G_{e} \cong(2.37 \underline{5276497}$ to $2.37 \underline{6043705}) \times 10^{37} \mathrm{~m}^{3} \mathrm{~kg}^{-1} \mathrm{sec}^{-2}$

$G_{N} \cong(6.67 \underline{0254668}$ to $6.67 \underline{456314}) \times 10^{-11} \mathrm{~m}^{3} \mathrm{~kg}^{-1} \mathrm{sec}^{-2}$

$\alpha_{S} \cong(0.1179 \underline{231391}$ to $0.1179 \underline{29483}) \cong 0.1185 \pm 0.0006$

\section{Characteristic atomic and nuclear applica- tions}

a) Fermi's weak coupling constant: It can be understood as follows [45]. 
$F_{W} \cong\left(\frac{m_{e}}{m_{p}}\right)^{2} \hbar c R_{0}^{2} \cong \frac{4 G_{s}^{2} m_{e}^{2} \hbar}{c^{3}}$

b) Root mean square radius of proton: It can be understood as follows[45], [48], [49], and [50].

$R_{p} \cong \frac{\sqrt{2} G_{s} m_{p}}{c^{2}}$

c) Bohr radius of electron: It can be understood as follows.

$a_{0} \cong\left(\frac{4 \pi \varepsilon_{0} G_{e} m_{e}^{2}}{e_{e}^{2}}\right)\left(\frac{G_{s} m_{p}}{c^{2}}\right)$

d) Proton-neutron beta stability line: It can be understood as follows [51].

$\left\{\begin{array}{l}\text { Let, } \\ k \cong\left(\frac{G_{s} m_{p} m_{e}}{\hbar c}\right) \cong\left(\frac{\hbar c}{G_{e} m_{e}^{2}}\right) \cong 1.6 \times 10^{-3}\end{array}\right.$

$A_{s} \cong 2 Z+k(2 Z)^{2}$

e) Nuclear binding energy at stable atomic nuclides: It can be understood as follows [51], [52]

For $(\mathrm{Z} \geq 5)$,

$$
\begin{aligned}
B E & \cong-\left(Z-2+\sqrt{\frac{Z}{30}}\right) \sqrt{\left(\begin{array}{c}
\frac{3}{5} \frac{e^{2}}{4 \pi \varepsilon_{0} R} \\
0 \quad p
\end{array}\right)\left(\begin{array}{c}
\frac{3}{5} \frac{s}{5} m^{2} \\
R
\end{array}\right)} \\
& \cong-\left(Z-2+\sqrt{\frac{Z}{30}}\right) \times 19.8 \mathrm{MeV}
\end{aligned}
$$

\section{Characteristic sub-nuclear applications}

RHIC have tentatively claimed to have created a quark-gluon plasma with an approximate temperature of 4 trillion degree Kelvin [53-56]. A new record breaking temperature was set by ALICE at CERN on August, 2012 in the ranges of 5.5 trillion degree Kelvin. In June 2015, an international team of physicists have produced quark-gluon plasma at the Large Hadron Collider by colliding protons with lead nuclei at high energy inside the supercollider's Compact Muon Solenoid detector at a temperature of 4 trillion degree Kelvin. With reference to the recommended up, down and strange quark masses, estimated geometric mean melting point is 14 trillion degree $\mathrm{K}$ and can be compared with the experimental results.

a) Melting point of proton: It can be understood as follows.

$$
T_{\text {proton }} \cong \frac{\hbar c^{3}}{8 \pi k_{B}\left(G_{s} m_{p}\right)} \cong 0.147 \text { Trillion } \mathrm{K}
$$

b) Melting point of electron: It can be understood as follows.

$$
T_{\text {electron }} \cong \frac{\hbar c^{3}}{8 \pi k_{B}\left(G_{e} m_{e}\right)} \cong 0.3786 \text { Million K }
$$

These two melting points are for experimental verification.

\section{Characteristic astrophysical applications}

a) Mass of neutron star: It can be understood as follows [57-61].

$$
\begin{aligned}
& \frac{G_{N} M_{N} m_{n}}{\hbar c} \cong \sqrt{\frac{G_{S}}{G_{N}}} \text { and } \\
& M_{N} \cong \sqrt{\frac{G_{s}}{G_{N}}}\left(\frac{\hbar c}{G_{N} m_{n}}\right)
\end{aligned}
$$

$\cong 3.17 \times$ Solar mass

where $\left(M_{N}, m_{n}\right)$ represent masses of neutron star and neutron respectively.

b) Radius of neutron star: It can be understood as follows.

c) Let $\left(R_{N}, R_{n}\right)$ represent the radii of neutron star [62] and neutron [63] respectively.

$$
\begin{aligned}
& \frac{R_{N}}{\left(R_{0} / 2\right)} \cong \sqrt{\frac{G_{s}}{G_{N}}} \\
& \rightarrow R_{N} \cong \sqrt{\frac{G_{s}}{G_{N}}}\left(\frac{R_{0}}{2}\right) \cong \sqrt{\frac{G_{S}}{G_{N}}}\left(\frac{G_{s} m_{n}}{c^{2}}\right) \cong 13.8 \mathrm{~km} \\
& \text { where } R_{n} \cong \frac{G_{s} m_{n}}{c^{2}} \cong 0.62 \mathrm{fm}
\end{aligned}
$$

\section{0. 'System of units' independent Avoga- dro number and Molar mass unit}

It is noticed that,

$$
\sqrt{\frac{G_{e}}{G_{N}}} \approx 5.96 \times 10^{23}
$$

In this context the authors could publish interesting contributions in Indian DAE-BRNS conference proceedings and International Intradisciplinary Conference on the Frontiers of Crystallography [36]. Even though, this is a semi empirical procedure, Avogadro number seems to be strongly connected with crystal structures as well as unification of fundamental forces. With this unified semi empirical procedure, it is possible to increase the scope and applicability of Avogadro number and with further research, independent of the 'gram mole' concept, absolute procedure for estimating the value of the Avogadro number can be developed. Proceeding further, the currently believed 'gram mole' or 'molar mass unit' [64-67] can be understood in the following way.

If 'atom as a whole' is believed to exhibit electromagnetic behavior, then

$G_{e}\left(m_{\text {atom }}\right)^{2} \cong G_{N}\left(M_{\text {mole }}\right)^{2}$

Where $m_{\text {atom }}$ is the unified atomic mass unit and $M_{\text {mole }}$ is the molar mass unit or gram mole.

Thus it is very clear to say that, directly and indirectly 'gravity' plays a key role in understanding the molar mass unit.

$$
\begin{aligned}
& \frac{M_{\text {mole }}}{m_{\text {atom }}} \cong \sqrt{\frac{G_{e}}{G_{N}}} \approx N_{A} \\
& \rightarrow M_{\text {mole }} \cong \sqrt{\frac{G_{e}}{G_{N}}} \times m_{\text {atom }}
\end{aligned}
$$


Independent of system of units, from this relation it may be possible to explore the physical meaning of the famous 'Gram mole' in a unified approach.

\section{Discussion}

It may be noted that,

1) Relations (5), (6), (7) and (22) clearly suggest the possible role of $\left(G_{N}, G_{e}\right)$ in nuclear and atomic physics.

2) Relations (9), (13), (14). (17) And (18) clearly suggest the possible role of $G_{s}$ in nuclear physics.

3) Relations (11) and (15) clearly suggest the combined role of $\left(G_{s}, G_{e}\right)$ in nuclear and atomic physics.

4) Relations (16) clearly suggest the possible role of $\left(G_{s}, G_{e}\right)$ in understanding the proton-neutron stability.

5) Relations (20) and (21) clearly suggest the possible combined role of $\left(G_{s}, G_{N}\right)$ in astrophysics.

6) Relations (19)clearly suggests the possible role of $G_{e}$ in sub-nuclear physics.

7) Relations (22) to (24) clearly suggest the possible role of $\left(G_{e}, G_{N}\right)$ in understanding atomic mass and molar mass unit in a unified approach independent of system of and units.

The authors would like to stress the fact that, with currently believed unified (main stream) physics models it is impossible to discover/fit/derive such relations. If one is willing to consider this fact as a real inadequacy of current unified physics models, the proposed two gravitational constants can be recommended for indepth study at fundamental level. From unification point of view, one can find many critical reviews on the foundations, predictions, current status and success of string theory in physics literature [6871].

Reiner Hedrichsays [71]: "String theory is at the moment the only advanced approach to a unification of all interactions, including gravity. But, in spite of the more than thirty years of its existence, it does not make any empirically testable predictions, and it is completely unknown which physically interpretable principles could form the basis of string theory. At the moment, "string theory" is no theory at all, but rather a labyrinthic structure of mathematical procedures and intuitions. The only motivations for string theory consist in the mutual incompatibility of the standard model of quantum field theory and of general relativity as well as in the metaphysics of the unification program of physics, aimed at a final unified theory of all interactions, including gravity".

Edward Witten says [72]:"Even though we do not really understand it, quantum gravity is supposed to be some sort of theory in which, at least from a macroscopic point of view, we average, in a quantum mechanical sense, over all possible space-time geometries. (We do not know to what extent this description is valid microscopically)."

In this context, it is very clear to say that, when a well believed theoretical model is failing in addressing the basic and practical problems connected with unification of general theory of relativity and quantum mechanics, first of all, it must be reviewed at fundamental level to have a well defined set of physical quantities and physical constants to proceed further for testable predictions at observable energy scales associated with elementary particles physics and astrophysics.

\section{Conclusion}

Proposed relations (5 to 24) clearly demonstrate the role of proposed gravitational constants assumed to be associated with proton and electron. At first sight, their physical existence appears to be ad-hoc, but by seeing the applications one may be forced to say that, there is 'some new physics' behind their assumed 'presence'.
Along with the proposed assumptions, key points and semi empirical relations, if one is willing to recall the old concepts which broadly falls in the category of 'strong gravity' as suggested by Abdus Salam, C.Civaram, K.P.Sinha, E.Recami, K. Tennakone, Usha Raut, V. De Sabbatta and Roberto Onofrio, everyone will be forced to consider the above relations for in-depth analysis at fundamental level.

Proceeding further, if one is willing to explore the possibility of incorporating the proposed assumptions either in String theory models or in Quantum gravity models, certainly, back ground physics assumed to be connected with proposed semi empirical relations can be understood and a 'practical' model of "everything" can be developed.

\section{References}

[1] W. Lerche. Recent Developments in String Theory. CERN-TH/97299. Proceedings of the XVIII International Symposium on Lepton Photon Interactions XVIII International Symposium on Lepton Photon Interactions, Hamburg, July 1997. arxiv:hep-th/9710246.

[2] Juan M. Maldacena. Gravity, Particle Physics and Their Unification. Int.J.Mod.Phys. A15S1 840-852 (2000)

[3] S. Schlamminger and R.D. Newman. Recent measurements of the gravitational constant as a function of time. Phys. Rev. D 91, 121101 (2015)http://dx.doi.org/10.1103/PhysRevD.91.121101.

[4] J. B. Fixler et al. Atom Interferometer Measurement of the Newtonian Constant of Gravity, Science 315 (5808): 74-77 (2007)http://dx.doi.org/10.1126/science.1135459.

[5] G. Rosi, et al. Precision measurement of the Newtonian gravitational constant using cold atoms. Nature 510, 518-521. (2014)http://dx.doi.org/10.1038/nature13433.

[6] Terry Quinn, Harold Parks, Clive Speake and Richard Davis. An uncertain big G.Phys.Rev. Lett. 112.068103. (2013)

[7] George $T$ Gillies. The Newtonian gravitational constant: recent measurements and related studies. Rep. Prog. Phys. 60 151, (1997)http://dx.doi.org/10.1088/0034-4885/60/2/001.

[8] J Stuhler, M Fattori, T Petelski and G M Tino. MAGIA using atom interferometry to determine the Newtonian gravitational constant. J. Opt. B: Quantum Semiclass. Opt. 5 S75-S81 (2003)http://dx.doi.org/10.1088/1464-4266/5/2/361.

[9] Roberto Onofrio. On Weak Interactions as Short- Distance Manifestations of Gravity. Modern Physics Letters A, Vol. 28, No. 7 1350022 (2013a) http://dx.doi.org/10.1142/S0217732313500223.

[10] Roberto Onofrio. Proton radius puzzle and quantum gravity at the Fermi scale. $\quad$ EPL 20002 (2013b)http://dx.doi.org/10.1209/0295-5075/104/20002.

[11] Abdus Salam. Strong Interactions, Gravitation and Cosmology. Publ. in: NATO Advanced Study Institute, Erice, June16-July 6, 1972.

[12] Salam A, Sivaram C. Strong Gravity Approach to QCD and Confinement. Mod. Phys. Lett., A8(4), 321- 326. (1993)http://dx.doi.org/10.1142/S0217732393000325.

[13] Recami E and V. T. Zanchin. The strong coupling constant: its theoretical derivation from a geometric approach to hadron structure. Foundations of Physics letters, vol-7, no.1, pp. 85-93.(1994).

[14] C. Sivaram and K. P. Sinha. Strong gravity, black holes, and hadrons. Phys. Rev. D 16 (1975).

[15] C. J. Ishame et al, f-Dominance of Gravity. Phys. Rev. D 3867. (1971)http://dx.doi.org/10.1103/PhysRevD.3.867.

[16] K. Tennakone. Electron, muon, proton, and strong gravity. Phys. Rev. D 10(1974) 1722http://dx.doi.org/10.1103/PhysRevD.10.1722.

[17] C. Sivaram et al. Gravitational charges, f-gravity and hadron masses. Pramana. Vol 2, No 5, pp229-238 (1974)http://dx.doi.org/10.1007/BF02847078.

[18] C. Sivaram and K. P. Sinha, Strong spin-two interaction and general Phys. Rep. 111(1979)http://dx.doi.org/10.1016/0370-1573(79)90037-1.

[19] C. Sivaram et al. Gravity of Accelerations on Quantum Scales and its consequences. http://arxiv.org/abs/1402.5071(2013)

[20] UshaRaut and K.P. Sinha. Strong gravity and the Fine structure constant. Proc. Indian Natn. Sci.Acad. 49 A, No 2, pp. 352-358 (1983) 
[21] K.P.Krishna. Gauge theories of weak and strong gravity. Pramana. Vol 23, No $2, \quad$ pp205-214 (1984)http://dx.doi.org/10.1007/BF02846517.

[22] V. De. Sabbata and C. Sivaram. Strong Spin-Torsion Interaction between Spinning Protons. IL NuovoCimento Vol. 101 A, No 2 pp.273-283, (1989)

[23] Recami E. Elementary Particles as Micro-Universes, and "Strong Black-holes": A Bi-Scale Approach to Gravitational and Strong Interactions. Preprint NSF-ITP- 02-94. Posted in the arXives as the eprint physics/0505149, and references therein.

[24] P. Caldirola, M. Pavsic and Recami E. Explaining the Large Numbers by a Hierarchy of Universes: A Unified Theory of Strong and Gravitational Interactions. IL NuovoCimento Vol. 48 B, No. 2, 11 (1978)

[25] V. T. Zanchin and Recami E. Regge like relations for stable (nonevaporating) black holes. Foundations of Physics letters, vol-7, no.2, pp.167-179 (1994).

[26] S. I. Fisenko, M. M. Beilinson and B. G. Umanov. Some notes on the concept of strong gravitation and possibilities of its experimental investigation. Physics Letters A, Vol-148, Issues 8-9, pp 405-407. (1990)

[27] M. Kumar and S. Sahoo. Elementary Particles as Black Holes and Their Binding Energies. The African Review of Physics 8:0025. pp. 165-168 (2013)

[28] David Gross, Einstein and the search for Unification. Current science, Vol. 89, No. 12, 25 (2005)

[29] U. V. S. Seshavatharam and S. Lakshminarayana. Nucleus in Strong nuclear gravity. Proceedings of the DAE Symp. On Nucl. Phys. 56: 302 (2011)

[30] U. V. S. Seshavatharam, Lakshminarayana S. Lakshminarayana. To Validate the Role of Electromagnetic and Strong Gravitational Constants via the Strong Elementary Charge. Universal Journal of Physics and Application 9(5): 210-219

(2015) http://dx.doi.org/10.13189/ujpa.2015.090503.

[31] U. V. S. Seshavatharam et al. On Fundamental Nuclear Physics \& Quantum Physics in Light of a Plausible Final Unification.Prespacetime Journal, Vol 6, Issue 12, pp. 1451-1468 (2015)

[32] U. V. S. Seshavatharam, Lakshminarayana S. To confirm the existence of nuclear gravitational constant, open Science Journal of Modern Physics. 2(5): 89-102 (2015)

[33] U. V. S. Seshavatharam, Lakshminarayana S. Final unification with Schwarzschild's Interaction. Journal of Applied Physical Science International 3(1): 12-22 (2015).

[34] U. V. S. Seshavatharam and S. Lakshminarayana. Analytical estimation of the gravitational constant with atomic and nuclear physical constants. Proceedings of the DAE-BRNS Symp. onNucl. Phys. 60: $850,(2015)$.

[35] U. V. S. Seshavatharam and S. Lakshminarayana. Understanding nuclear structure with Schwarzschild interaction and Avogadro number. Proceedings of the DAE-BRNS Symp. OnNucl. Phys. 60 "852 (2015)

[36] U. V. S. Seshavatharam and S. Lakshminarayana. Semi empirical procedure for estimating the Newtonian gravitational constant with elementary physical constants and Avogadro number. Proceedings of International Intradisciplinary Conference on the Frontiers of Crystallography (IICFC-2014). pp.47- 60 (2015)

[37] U. V. S. Seshavatharam and S. Lakshminarayana. Applications of gravitational model of possible final unification in both large and small scale physics. Prespacetime journal, Vol 7, issue2, 300-316 (2016).

[38] Roger Penrose. Chandrasekhar, Black Holes, and Singularities. J. Astrophys. Astr. 17, pp. 213 231(1996)http://dx.doi.org/10.1007/BF02702305.

[39] Subrahmanyan Chandrasekhar. On Stars, Their Evolution and Their Stability. Nobel Prize lecture, (8 December 1983).

[40] G W Gibbons. The Maximum Tension Principle in General relativity. Found.Phys.32:1891-1901 (2002) http://dx.doi.org/10.1023/A:1022370717626.

[41] Michael R.R. Good and Yen Chin Ong. Are Black Holes Springy? Phys. Rev. D $041, \quad 04031 \quad$ (2015) http://dx.doi.org/10.1103/PhysRevD.91.044031.

[42] Hawking, S.W. Particle Creation by Black Holes. Commun.Math Phys., 43: 199-220 (1975).http://dx.doi.org/10.1007/BF02345020.

[43] AbhasMitra. Why gravitational contraction must be accompanied by emission of radiation in both Newtonian and Einstein gravity. Phys. Rev. D 74, (2006)http://dx.doi.org/10.1103/PhysRevD.74.024010.
[44] AbhasMithra and K.K.Singh. The mass of the OppenheimerSynder- black hole: only finite mass quasi-black holes. International Journal of Modern Physics D Vol. 22, No. 9 (2013).

[45] K.A. Olive et al. (Particle Data Group), Chin. Phys. C, 38, 090001 (2014)http://dx.doi.org/10.1088/1674-1137/38/9/090001.

[46] Geiger H, Marsden E. On a diffuse reaction of the particles. Proc. Roy. Soc., $\quad$ Ser. A $\quad$ A : 495-500, (1909)http://dx.doi.org/10.1098/rspa.1909.0054.

[47] H. Yukawa. On the Interaction of Elementary Particles. Proc. Phys. Math. Soc. Jap. 17 (48) (1935)

[48] Robert Hofstadter, Rudolf Mössbauer. The electron-scattering method and its application to the structure of nuclei and nucleons. Nobel Lecture, December 11, (1961)

[49] P.J. Mohr, B.N. Taylor, and D.B. Newell. CODATA Recommended Values of the Fundamental Physical Constants: 2010" by in Rev. Mod. Phys. 84, $1527 \quad$ (2012) http://dx.doi.org/10.1103/RevModPhys.84.1527.

[50] CE.Carlson.The proton radius puzzle. Accepted invited review for Progress in Particle and Nuclear Physics.http://arxiv.org/pdf/1502.05314.pdf.

[51] Chowdhury, P.R. et al. Modified Bethe-Weizsacker mass formula with isotonic shift and new driplines. Mod. Phys. Lett. A20 p.16051618. (2005).http://dx.doi.org/10.1142/S021773230501666X.

[52] N.Ghahramany et al. New approach to nuclear binding energy in integrated nuclear model. Journal of Theoretical and Applied Physics 6:3 (2012).http://dx.doi.org/10.1186/2251-7235-6-3.

[53] V. Khachatryan et al. (CMS Collaboration), Evidence for Collective Multiparticle Correlations in $\mathrm{p}-\mathrm{Pb}$ Collisions, Phys. Rev. Lett. 115 012301 (2015)http://dx.doi.org/10.1103/PhysRevLett.115.012301.

[54] ATLAS Collaboration, Observation of Associated Near-Side and Away-Side Long-Range Correlations in $\sqrt{s_{N N}}=5.02 \mathrm{TeV}$ ProtonLead Collisions with the ATLAS Detector, Phys. Rev. Lett. 110, 182302 (2013)http://dx.doi.org/10.1103/PhysRevLett.110.182302.

[55] CMS Collaboration, Multiplicity and transverse momentum dependence of two- and four-particle correlations in $\mathrm{pPb}$ and $\mathrm{PbPb}$ collisions, Phys. Lett. B 724,213 (2013)http://dx.doi.org/10.1016/j.physletb.2013.06.028.

[56] W.A. Zajc .The Fluid Nature of Quark-Gluon Plasma. Nucl.Phys.A805:283-294, (2008)http://dx.doi.org/10.1016/j.nuclphysa.2008.02.285.

[57] Chandrasekhar, S. The Highly Collapsed Configurations of a Stellar Mass. Monthly Notices of the Royal Astronomical Society, 95, 207 225 (1935)http://dx.doi.org/10.1093/mnras/95.3.207.

[58] Bombaci, I. The Maximum Mass of a Neutron Star. Astronomy and Astrophysics, 305, 871-877. (1996)

[59] Srinivasan, G. The Maximum Mass of Neutron Stars. Bulletin of Astronomic Society of India, 30, 523-547. (2002) http://dx.doi.org/10.1007/s001590200016.

[60] James M. Lattimer. The Nuclear Equation of State and Neutron Star Masses. Annu. Rev. Nucl. Part. Sci. 62:485-515 (2012)http://dx.doi.org/10.1146/annurev-nucl-102711-095018.

[61] N. Chamel et al. On the Maximum Mass of Neutron Stars. Int. J. $\begin{array}{llll}\text { Mod. } & \text { Phys. } 22, & 1330018 & \text { (2013) }\end{array}$ http://dx.doi.org/10.1142/S021830131330018X

[62] SebastienGuillot et al. Measurement of the Radius of Neutron Stars with High S/N Quiescent Low-mass X-ray Binaries in Globular Clusters. Astrophys.J. 772 (2013).http://dx.doi.org/10.1088/0004$\underline{637 x / 772 / 1 / 7}$.

[63] S. Abrahamyan et al. Measurement of the Neutron Radius of $208 \mathrm{~Pb}$ through Parity-Violation in Electron. Phys. Rev. Lett. 108, 112502 (2012) http://dx.doi.org/10.1103/PhysRevLett.108.112502.

[64] Martin J. T. Milton. A new definition for the mole based on the Avogadro constant: a journey from physics to chemistry. Phil. Trans. R. Soc. A 369, 3993-4003 (2011) (2011)

[65] Giancarlo D'Agostino ET AL. 30Si Mole Fraction of a Silicon Material Highly Enriched in 28Si Determined by Instrumental Neutron Activation Analysis. Anal. Chem., 87 (11), pp 5716-5722 (2015)http://dx.doi.org/10.1021/acs.analchem.5b00878.

[66] K.A. Bronnikov et al. On new definitions of SI base units. Why is the "atomic" kilogram preferable? arxiv:1410.7906 (2014)

[67] P. Fraundorf and Melanie Lipp. A graphite-prism definition for Avogadro's “integer". arxiv: $1201.5537 \mathrm{v} 4$. (2015)

[68] Lee Smolin. Trouble with physics. The rise of the string theory, the fall of science. New York: Houghton Mifflin Co. (2006)

[69] Peter Woit. Not Even Wrong: The Failure of String Theory and the Search for Unity in Physical Law. London: Jonathan Cape \&: New York: Basic Books.(2006) 
[70] Penrose, Roger. The Road to Reality: A Complete Guide to the Laws of the Universe. Knopf. (2005)

[71] Reiner Hedrich. The Internal and External Problems of String Theory. A Philosophical View. J.Gen.Phil.Sci.38:261-278 (2007)http://dx.doi.org/10.1007/s10838-007-9048-3.

[72] Edward Witten. What Every Physicist Should Know About String Theory. GR Centennial Celebration, Strings 2015, Bangalore, India (2015).

http://member.ipmu.jp/yuji.tachikawa/stringsmirrors/2015/26-06-

2015-Edward-Witten.pdf. 